\title{
Probabilistic Modelling of Microstructural Evolution in Zr Based Bulk Metallic Glass Matrix Composites during Solidification in Additive Manufacturing
}

\author{
Muhammad Musaddique Ali Rafique \\ Eastern Engineering Solutions LLC, Detroit, MI, USA \\ Email: ali.rafique@hotmail.com
}

How to cite this paper: Rafique, M.M.A. (2018) Probabilistic Modelling of Microstructural Evolution in Zr Based Bulk Metallic Glass Matrix Composites during Solidification in Additive Manufacturing. Engineering, 10, 130-141. https://doi.org/10.4236/eng.2018.104010

Received: February 11, 2018

Accepted: April 10, 2018

Published: April 13, 2018

Copyright $\odot 2018$ by author and Scientific Research Publishing Inc. This work is licensed under the Creative Commons Attribution International License (CC BY 4.0).

http://creativecommons.org/licenses/by/4.0/ (c) (i) Open Access

\begin{abstract}
Bulk metallic glass and their composites (BMGMCs) are a new class of materials which possess superior mechanical properties as compared to existing conventional materials. Owing to this, they are potential candidates for tomorrow's structural applications. However, they suffer from poor ductility and little or no toughness which render them brittle and they manifest catastrophic failure under applied force. Their behavior is dubious, unpredictable and requires extensive experimentation to arrive at conclusive results. In present study, an effort has been made to design bulk metallic glass matrix composites by the use of modeling and simulation. A probabilistic cellular automaton (CA) model is developed and described in present study by author which is used in conjunction with earlier developed deterministic model to predict microstructural evolution in $\mathrm{Zr}$ based BMGMCs in additive manufacturing liquid melt pool. It is elaborately described with an aim to arrive at quantitative relations which describe process and steps of operations. Results indicate that effect of incorporating all mass transfer and diffusion coefficients under transient conditions and precise determination of probability number play a vital role in refining the model and bringing it closer to a level that it could be compared to actual values. It is shown that proposed tailoring can account for microstructural evolution in metallic glasses.
\end{abstract}

\section{Keywords}

Bulk Metallic Glass Matrix Composites, Solidification, Cellular Automata Method 


\section{Introduction}

Bulk metallic glass matrix composites [1] are unique materials of future having superior mechanical properties which surpasses any other material known till date. Their high hardness, strength and elastic strain limit make them potential candidates for future extreme engineering applications [2]-[8]. However, they suffer from little or no toughness under tensile loading. In fact, they exhibit tensile softening. This is a detrimental property which renders them unusable for any practical use. This can be overcome by various means. One of which is in-situ introduction of primary phase ductile particles (e.g. spheroidal B2-CuZr intermetallic) during solidification which serves as an obstacle to movement of shear band thus pin them and increase their toughness. This phenomenon is difficult to control, and a lot of rigorous experimentations are required before conclusive results could be drawn. In present study, which is continuation of author's previous work [2], a probabilistic model of nucleation and growth [9] [10] is developed to supplement deterministic model to describe this phenomena in these alloys without recurrence to experimentation. Much research has been carried out previously on modelling and simulation of solidification phenomena in various processes employing cellular automata or modified cellular automata combined with finite element such as modified additive manufacturing (HDMR [11], LAMP [12] on 316L SS [10], two dimensional CAFE [13], cladding [14] [15]), selective laser melting (SLM) using CAFE [14] [15] [16] [17], CAPF, CAFVM [18] and modified CAFE [19] but none has been conducted on the use of cellular automata method on bulk metallic glasses or their composites. On modelling and simulation of bulk metallic glasses themselves; only two studies have been reported [20] [21] but again they are not aimed at explaining microstructure evolution. At present scenario, there is no single hybrid/combined model which explains phenomena of heat transfer (liquid melt pool formation as a result of laser-matter interaction and its evolution-solidification) coupled with mass transfer (nucleation and growth (solute diffusion [22] and capillary action driven)) to predict microstructure and grain size in bulk metallic glass matrix composites as melt cools in additive manufacturing liquid melt pool. Present study is one step forward to answer second (i.e. microscale mass transfer) question. Aim is to model nucleation of primary phase ductile crystalline particles in glassy matrix. Constitutive equations governing microscale mass transfer and diffusion phenomena in individual cell of cellular automata grid are developed which describe evolution of solid fraction and solid fraction increment in each cell as a function of time. Together with neighbourhood transition rules, this will give visualization of microstructure evolved in each cell. Model is described as follows.

\section{Model}

In order to formulate model in present case, following assumptions are drawn. 


\subsection{Assumptions}

1) Model is developed under steady state conditions.

2) Regular square shape is chosen as shape of one cell out of square, hexagon or triangle.

3) Decentred rectangular shape is chosen to counteract mesh anisotropy.

4) One of crystallographic orientations (e.g. one of $<100\rangle$ directions for fcc metals) of grain stands perpendicular to simulation plane. This direction is diagonal of square and labelled simply as $<10>$ direction (Figure 1 ).

5) Incubation time (time in which active side branches are influenced by solute field of their neighbours) is neglected.

6) Nucleation radius of grains is neglected.

A summary of steps to be followed by cellular automaton simulation is described below;

1) Choose regular shape for populating cellular automaton (CA) grid (e.g. square).

2) Define decentred square envelope.

3) Grow grain in it under steady state conditions.

4) Grain A nucleates at centre of cell $v$ (it has inherent/intrinsic misorientation of its crystallographic axis to that of $\mathrm{CA}$ axis).

NOTE: Misorientation in present case is intrinsic feature (i.e. it is caused by features of grains). To remove it, either choose different lattice (hexagonal, octagonal) "or" refine the algorithm.

In reality, this growth of dendritic front has to be tackled at the level of secondary dendritic arm spacing. This may be done by two ways;

1) Introducing dendrite tip correction or

2) Use of $2 \mathrm{D}$ rectangular algorithm [9] at secondary dendrite arm spacing level.

Both these approaches are difficult to implement and practice as "first" is not a CA model at all while "second" is difficult to extend to 3D as rectangle would correspond to non-regular octahedron shape. That's why, a new approach known as $2 \mathrm{D}$ decentred square growth algorithm is developed and adopted (present study) (Figure 2).

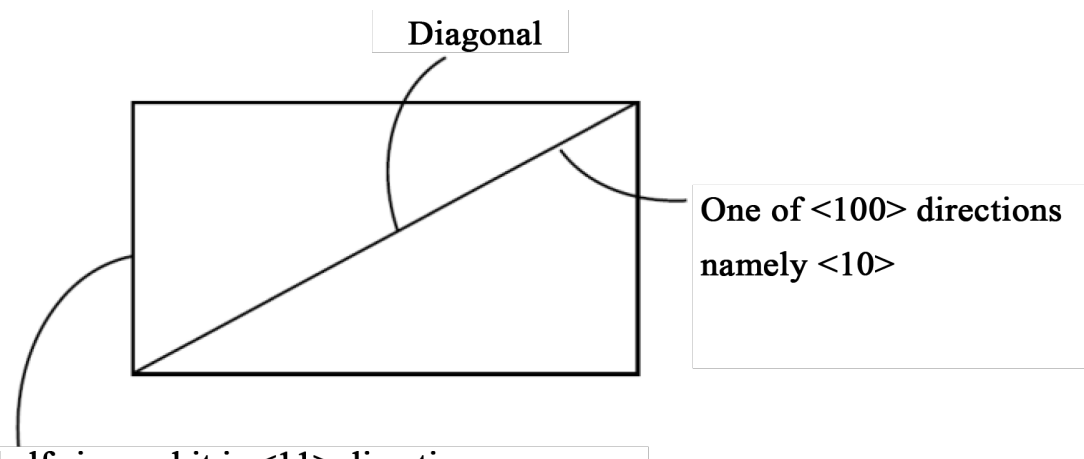

for half size and it is $<11>$ direction

Figure 1. Schematic of crystallographic orientation of one direction. 


\subsection{Procedure of Cellular Automaton Model}

Cellular automaton model consists of [24] discretizing

1) Simulated area into finite cells while

2) Time into small time steps

At a certain time, a cell has a "state" that can be described by number of variables. Most important of which are;

1) Temperature and

2) Solute concentration

During simulation, the state of each cell is determined by the state of its nearest neighbors through a transition rule known as neighborhood transition rule. Two types of neighborhood transition rules are commonly employed.

1) Moore's law (8 neighborhood)

2) Von Neuman's law (4 neighborhood)

In traditional CA models, the state of all cells is simultaneously updated. Cell switch states depending on the outcome of the transition rules. In present case, Von Neumann neighborhood is used (4 nearest neighbors). CA rule of Von Neumann neighborhood can be determined by

$$
a_{i j}^{t+\Delta t}=\varnothing\left(a_{i-1 j}^{t}+a_{i j-1}^{t}+a_{i j}^{t}+a_{i j+1}^{t}+a_{i+1 j}^{t}\right)
$$

where $a_{i j}^{t}$ is state of cell, which has mark number $(i, j)$ at the time $t ; \varnothing$ is the evolvement rule and it is vital that this rule is carefully chosen to reflect the objective physics of the simulated phenomena. It may be chosen out of 256 rules.

Now, solute diffusion is explained for spheroidal B2-CuZr intermetallic in glassy matrix. This solute diffusion happens in two steps a) Solute diffusion in single cell and b) Solute diffusion between cells.

\subsubsection{Solute Diffusion in Single Cell}

At a particular time, each CA cell may have three possible states; liquid (solid fraction is 0 ), mushy (solid fraction between 0 and 1 ), or solid (solid fraction is 1). Cell state changes from liquid to mushy when nucleation occurs. Solute diffusion in a cell containing "liquid" and "solid" are described by [11] [23];

$$
\frac{\partial C_{L}}{\partial t}=\frac{\partial}{\partial x}\left[D_{L} \frac{\partial C_{L}}{\partial t}\right]+\frac{\partial}{\partial y}+\left[D_{L} \frac{\partial C_{L}}{\partial y}\right]+C_{L}(1-k) \frac{\partial f_{s}}{\partial t}
$$

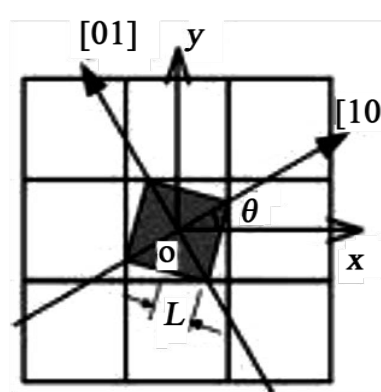

(a)

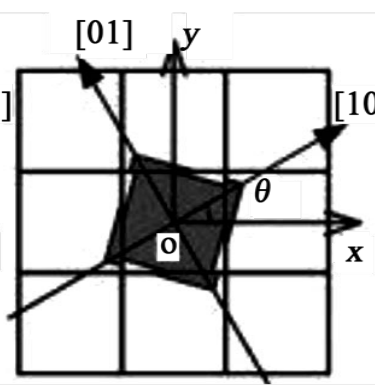

(b)

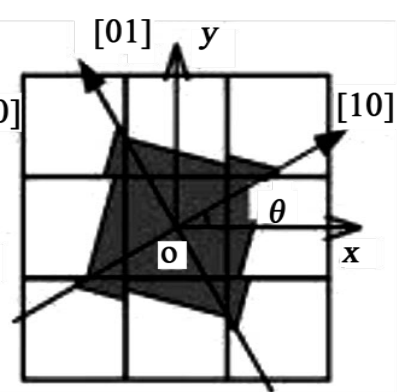

(c)

Figure 2. Schematic of modified 2D decentred square CA growth algorithm [23]. 
and

$$
\frac{\partial C_{s}}{\partial t}=\frac{\partial}{\partial x}\left[D_{s} \frac{\partial C_{s}}{\partial x}\right]+\frac{\partial}{\partial y}\left[D_{s} \frac{\partial C_{s}}{\partial y}\right]
$$

respectively, where $C_{L}$ is solute concentration in liquid, $C_{S}$ is same in solid and $D_{L}$ is solute diffusion coefficient in liquid and $D_{S}$ is same in solid. Similarly, solute diffusion in a mushy cell is given by below equations

$$
\begin{gathered}
\frac{\partial C_{E}}{\partial t}=\nabla \times\left(D_{E} \nabla \times C_{E}\right) \\
C_{E}=C_{L}\left(1-f_{s}\right)+C_{s} f_{s} \\
D_{E}=D_{L}\left(1-f_{s}\right)+C_{s} f_{s} k
\end{gathered}
$$

where $D_{L}$ and $D_{s}$ are the diffusion coefficients of the liquid phase and solid phase, respectively. $f_{s}$ is the solid fraction, $C_{E}$ is the equivalent concentration, and $D_{E}$ is equivalent diffusion coefficient.

1) Fraction Solid in Single Cell

To calculate solid fraction increment, first growth velocities of solid liquid interface in $\mathrm{x}$ and $\mathrm{y}$ directions are calculated [25] [26]. This is briefly described below;

$$
\begin{aligned}
& V_{x}(i, j) \\
& =\frac{D_{L}}{a(1-k)}\left[\left(1-\frac{C_{L}(i-1, j)}{C_{L}^{*}(i, j)}\right) f_{L}(i-1, j)+\left(1-\frac{C_{L}(i+1, j)}{C_{L}^{*}(i, j)}\right) f_{L}(i+1, j)\right] \\
& +\frac{k D_{s}}{a(1-k)}\left[\left(1-\frac{C_{s}(i-1, j)}{k C_{L}^{*}(i, j)}\right) f_{s}(i-1, j)+\left(1-\frac{C_{s}(i+1, j)}{k C_{L}^{*}(i, j)}\right) f_{s}(i+1, j)\right] \\
& V_{y}(i, j) \\
& =\frac{D_{L}}{a(1-k)}\left[\left(1-\frac{C_{L}(i, j-1)}{C_{L}^{*}(i, j)}\right) f_{L}(i, j-1)+\left(1-\frac{C_{L}(i, j+1)}{C_{L}^{*}(i, j)}\right) f_{L}(i, j+1)\right] \\
& +\frac{k D_{s}}{a(1-k)}\left[\left(1-\frac{C_{s}(i, j-1)}{k C_{L}^{*}(i, j)}\right) f_{s}(i, j-1)+\left(1-\frac{C_{s}(i, j+1)}{k C_{L}^{*}(i, j)}\right) f_{s}(i, j+1)\right]
\end{aligned}
$$

once calculated, fraction solid evolution is calculated by

$$
\delta_{f_{s}}=\frac{\delta t}{a}\left[V_{x}+V_{y}+V_{x} V_{y} \frac{\delta t}{a}\right]
$$

where $a=$ mesh size (uniform and constant) for both $x$ and $y$ direction and $\delta t$ is time step.

In a single CA cell, solid fraction can be calculated using

$f_{s}=f_{s}^{o}+\delta f_{s}$

where $f_{s}^{o}=$ solid fraction at previous time step

Time step itself may be calculated by

$$
\delta t=\frac{1}{5} \min \left(\frac{\Delta x}{V_{\max }}, \frac{\Delta x^{2}}{D_{L}}, \frac{\Delta x^{2}}{D_{s}}\right)
$$


where $\Delta x$ is cell size, and $V_{\max }$ is maximum growth velocity of cell during each time step.

Note: solid fraction $\left(f_{s}\right)$ and solid fraction increment $\left(\delta_{f_{s}}\right)$ are two different things and should not be confused. Former means actual numerical increase in amount of solid evolved in a cell during time step $\delta t$ while later is difference of value between old and new.

2) Mean Curvature of Solid Liquid Interface

By a similar procedure, mean curvature of solid liquid (S/L) interface $k$ may be determined

$k=\frac{1}{a}\left[1-\frac{2}{N+1}\left(f_{s}+\sum_{i=1}^{N} f_{s}^{i}\right)\right]$

where $N$ is the neighboring cells and $f_{s}$ is the solid fraction

$$
T_{\text {liq }}^{o}-T_{\text {local }}=\Delta T_{c}-\Delta T_{k}
$$

where $T_{\text {liq }}^{o}$ is liquidus temperature at $C_{o}, T_{\text {local }}$ is local temperature and $\Delta T_{c}, \Delta T_{k}$ are constitutional and curvature under cooling temperatures respectively.

Consider $\Delta T_{c}=-m_{L}\left(C_{L}^{*}-C_{o}\right)$ and $\Delta T_{k}=\Gamma_{k}$

Putting in (2)

$$
\begin{gathered}
T_{\text {liq }}^{o}-T_{\text {local }}=-m_{L}\left(C_{L}^{*}-C_{o}\right)-\Gamma_{k} \\
-m_{L}\left(C_{L}^{*}-C_{o}\right)=T_{\text {liq }}^{o}-T_{\text {local }}+\Gamma_{k} \\
\left(C_{L}^{*}-C_{o}\right)=-\frac{T_{\text {liq }}^{o}-T_{\text {local }}+\Gamma_{k}}{m_{L}} \\
\left(C_{L}^{*}-C_{o}\right)=-\frac{1}{m_{L}}\left(T_{\text {liq }}^{o}-T_{\text {local }}+\Gamma_{k}\right) \\
C_{L}^{*}=C_{o}-\frac{1}{m_{L}}\left(T_{\text {liq }}^{o}-T_{\text {local }}+\Gamma_{k}\right)
\end{gathered}
$$

where $C_{o}=$ initial concentration, $m_{L}=$ liquidus slope, $\Gamma_{k}=$ Gibbs Thomson coefficient, $k=$ mean curvature of S/L interface.

\subsubsection{Solute Diffusion between Cells}

Procedure adopted to calculate solute diffusion between cells is similar to the one adopted for calculation of solute diffusion in a single cell. Primarily, diffusion occurs owing to concentration gradient. This gradient is created by the release of solute in both liquid and solid. This may be summarized in following four steps [24];

Step 1: During this equilibrium at solid liquid interface is determined by $C_{s}^{*}=k C_{L}^{*}$

where $C_{s}^{*}$ is solute concentration in solid at solid/liquid interface. $C_{L}^{*}$ is counterpart in liquid and $k$ is partition coefficient. It is utmost important to de- 
termine $k$ in a proper manner preferably keeping in view transient conditions such that actual process conditions are simulated.

Step 2: Partitioning of solute in liquid is determined. This may be done by the use of equation 1 by replacing all subscripts by $L$.

Step 3: Partitioning of solute in solid is determined by again using Equation 1 without replacing any subscript or term.

Step 4: Partitioning of solute in growing cell may be determined as follows;

Neighbors of a cell could be anything between liquid, solid or growing. For a liquid cell, it could be solid or growing. For a solid cell, it could be liquid or growing and for a growing cell, it could be liquid, solid or even growing. When the computing center is a growing cell, regardless of state (i.e. solid, liquid or growing) of the neighbor of growing cell, the solute concentration of growing cell may be expressed as an equivalent concentration $C_{E}$, which may be defined as;

$$
C_{E}=C_{L}\left(1-f_{s}\right)+C_{s} f_{s}=C_{L}\left[1-(1-k) f_{s}\right]
$$

when $f_{s}=1, C_{E}=C_{S}$ and when $f_{s}=0, C_{E}=C_{L}$

Thus, diffusion equation may be expressed as;

$$
\frac{\partial C_{E}}{\partial t}=\nabla\left(D_{E} \nabla C_{E}\right)
$$

where $C_{E}$ is equivalent concentration defined by above equation and $D_{E}$ is equivalent diffusion coefficient [23]. This is schematically represented in Figure 3 below.

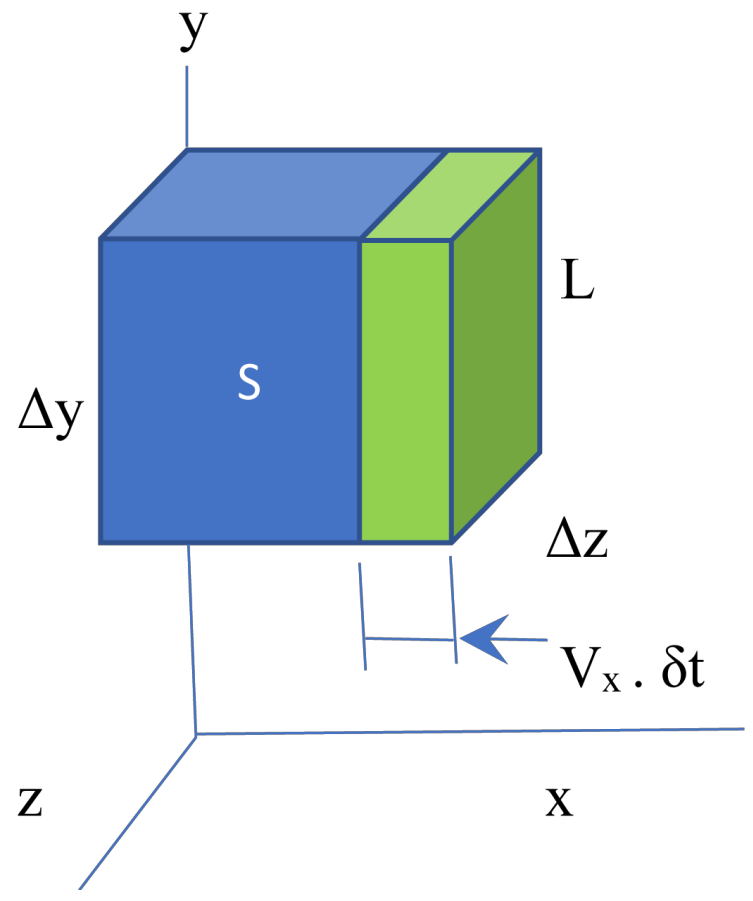

Figure 3. Schematic diagram of growing in a cell. 


\subsection{Interpolation}

Since thermal calculations are done at finite element mesh. There is need to relate finite element mesh to cellular automaton mesh. This is done by defining interpolation equation [27].

Physical domain in three-dimensional space is divided into cells of equal size $0.1 \mu \mathrm{m} \times 0.1 \mu \mathrm{m} \times 0.1 \mu \mathrm{m}$. Each cell is characterized by three variables.

1) State (solid, mushy cell or liquid)

2) Temperature and

3) Crystallography

Temperature values are calculated on the macro nodes and interpolation equation is needed for use in CA cell. This equation may be described as

$$
\begin{aligned}
T_{C A} & =\frac{\sum_{i=1}^{8} D_{i} T_{F E}}{\sum_{i=1}^{8} D_{i}} \\
D_{i} & =\exp \left(\frac{1}{d}\right)
\end{aligned}
$$

where $d=$ distance between 8 nearest nodes (moors model) in finite element model and center of cellular automaton cell (Figure 4).

At the start of simulation, all cells states are considered solid and all crystallographic variables are zero (non-active cells). As simulation starts, temperature of each cell is given by thermal simulation performed on finite element mesh nodes.

If temperature of cell passes the melting temperature $\left(T_{m}\right)$, its state changes to liquid. If it does not pass $T_{m}$, it stays as solid and if it stays at $T_{m}$, it becomes mushy.

Calculation of Nucleation Density of New Cells and Assigning p Number

Solute diffusion is calculated only for cells which experience melting and solidification (active cells). Liquid cell can again become solid if one of following condition happens. Nucleation or Capturing by another active-solid CA cells.

In each time step density of new nuclei [10] is determined by equation

$$
\frac{\mathrm{d} n}{\mathrm{~d}(\Delta T)}=\frac{n_{\max }}{\sqrt{2 \pi}} \exp \left[-\frac{\left(\Delta T-T_{\max }^{o}\right)}{2 \Delta T_{\sigma}^{2}}\right]
$$

This equation is tailored to take into account nucleation occurring at

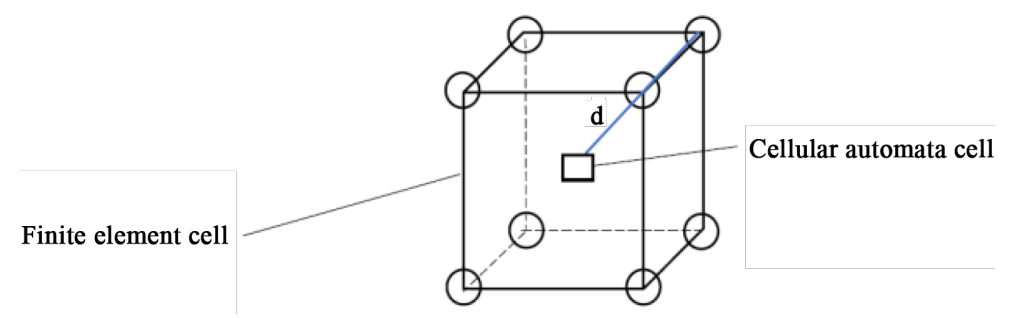

Figure 4. Representation of interpolation in one cell. 
1) Molten pool wall and

2) Bulk liquid

Nucleation densities are then multiplied by the total number of cells for Molten pool wall and bulk liquid in order to calculate number of new nucleation sites

$$
N_{s}=\frac{n_{\max }}{\sqrt{2 \pi}} \exp \left[-\frac{\left(\Delta T-\Delta T_{\max }^{o}\right)^{2}}{2 \Delta T_{s \sigma}^{2}}\right] N_{a s}
$$

where $N_{s}=$ Number of new nucleation sites at the molten pool wall and $N_{a s}$ $=$ Total number of liquid cells at the molten pool wall. Similarly

$$
N_{v}=\frac{n_{\max }}{\sqrt{2 \pi}} \exp \left[-\frac{\left(\Delta T-\Delta T_{\max }^{o}\right)^{2}}{2 \Delta T_{v \sigma}^{2}}\right] N_{a v}
$$

where $N_{v}=$ Number of new nucleation sites inside bulk liquid and $N_{a v}=$ Total number of liquid cells inside bulk liquid.

After calculating the number of new nucleation sites, a random number $(0<P$ $<1$ ) is assigned to each liquid cell. The nucleas is only formed in a cell $i$ which is located at molten pool wall if following condition is satisfied.

$$
P_{i}=\frac{N_{i}}{N_{a s}}
$$

Similarly, this condition must be satisfied for cells located in bulk liquid. This ensures that state of cell $i$ changes from liquid to solid as nucleation occurs whether it is at molten pool boundary or inside bulk liquid.

Another important parameter necessary to describe probabilistic phenomena is assigning of "preferential growth orientation". Again, this is determined by a random process. Certain numbers of growth orientations are chosen in three-dimensional spherical space around each nucleated cell as possible growth orientation. State of cell $i$ is chosen randomly from these orientations as nucleation occurs. Different growth velocities are attained by different grains because they have different preferential growth direction. A parameter known as orientation weight coefficient $\left(W_{i}^{j}\right)$ account for this effect. The growth length of cell $i$ with regards to its liquid neighbor $j$ (one of possible 26 cells) at time $t_{c}$ can be calculated by:

$$
l_{i}^{j}\left(t_{c}\right)=W_{i}^{j} \int_{0}^{t_{c}} v_{g} \mathrm{~d} t
$$

where $W_{i}^{j}$ is the orientation weight coefficient related to angle between preferential growth direction of cell $i$ and vector from cell $i$ linking to cell $j$ (called vector $L_{i}^{j}$ ). Orientation weight coefficient itself may be calculated by [28];

$$
W_{i}^{j}=\operatorname{Max}\left[\left|X_{w}\right|,\left|Y_{w}\right|,\left|Z_{w}\right|\right]
$$

where $X_{W}, Y_{W}$ and $Z_{W}$ may be calculated by solving below matrix

$$
\left[\begin{array}{c}
X_{w} \\
Y_{w} \\
Z_{w}
\end{array}\right]=\left[\begin{array}{ccc}
x_{p} & x_{q} & x_{r} \\
y_{p} & y_{q} & y_{r} \\
z_{p} & z_{q} & z_{r}
\end{array}\right] \cdot\left[\begin{array}{c}
P_{i}^{j} \\
q_{i}^{j} \\
r_{i}^{j}
\end{array}\right]
$$


where $\left(x_{p}, x_{q}, x_{r}\right),\left(y_{p}, y_{q}, y_{r}\right)$ and $\left(z_{p}, z_{q}, z_{r}\right)$ are direction cosines of [100] and [010] and [001] dendrite arms relating to the coordinate $x, y, z$ axes respectively and $\left(p_{i}^{j}, q_{i}^{j}, r_{i}^{j}\right)$ are direction cosines of vector $L_{i}^{j}$ relating to the coordinate $x, y, z$ axes respectively.

In detail, $l_{i}^{j}$ is growth length between two points $i$ and $j$ ( $i$ has solid state and $j$ has liquid state) when crystallographic orientation of cell $i$ is considered. Neighbor $j$ is captured by cell $i$ if below condition is satisfied.

$$
\frac{l_{i}^{j}\left(t_{c}\right)}{L} \geq 1
$$

where $L=l_{\text {cell }}$ if $j$ is one of 6 nearest neighbors, $L=\sqrt{2} l_{\text {cell }}$ if $j$ is one of twelve second-nearest neighbors, and $L=\sqrt{3} l_{\text {cell }}$ if $j$ is one of eight third-nearest neighbors.

If cell $j$ is captured by $i$, it will be assigned same orientation as cell $i$. Interested reader may find further detail in [28].

\section{Conclusion}

In present study, a cellular automata method is described for describing nucleation and growth of primary ductile phase particles in glassy matrix in bulk metallic glass matrix composites. Model is built on constitutive equations of microscale mass transfer and diffusion phenomena. Model is built assuming steady state conditions. However, use of transient conditions is recommended and as it brings model and thus simulation results to actual values. Coupling of finite element and cellular automata method is described by the help of interpolation equations. It is found that proper use of transport equations and calculations of random probability number play pivotal role in describing microscale solute diffusion and solid fraction evolution in solidifying alloy. Use of moderate size simulation grid (cartesian) to counter mesh anisotropy along with selection of decentered square algorithm also helps in model refinement and optimization.

\section{References}

[1] Qiao, J., Jia, H. and Liaw, P.K. (2016) Metallic Glass Matrix Composites. Materials Science and Engineering: R: Reports, 100, 1-69.

https://doi.org/10.1016/j.mser.2015.12.001

[2] Rafique, M.M.A., Qiu, D. and Easton, M. (2017) Modeling and Simulation of Microstructural Evolution in Zr Based Bulk Metallic Glass Matrix Composites during Solidification. MRS Advances, 2, 3591-3606.

https://www.cambridge.org/core/journals/mrs-advances/article/modeling-and-simu la-

tion-of-microstructural-evolution-in-zr-based-bulk-metallic-glass-matrix-composit es-during-solidification/E63B55899589FE3009C114E8B7C7430D

[3] Roberts, S.N. (2014) Developing and Characterizing Bulk Metallic Glasses for Extreme Applications. California Institute of Technology, Pasadena.

[4] Hofmann, D.C. and Roberts, S.N. (2015) Microgravity Metal Processing: From Undercooled Liquids to Bulk Metallic Glasses. Npj Microgravity, 1, 15003. https://doi.org/10.1038/npjmgrav.2015.3 
[5] Davidson, M., et al. (2013) Investigating Amorphous Metal Composite Architectures as Spacecraft Shielding. Advanced Engineering Materials, 15, 27-33. https://doi.org/10.1002/adem.201200313

[6] Kaufman, L., Perepezko, J. and Hildal, K. (2007) Synthesis and Performance of Fe-Based Amorphous Alloys for Nuclear Waste Repository Applications. Lawrence Livermore National Laboratory (LLNL), Livermore.

[7] Ojovan, M. and Batyukhnova, O. (2007) Glasses for Nuclear Waste Immobilization. WM07 Conference, 25 February-1 March 2007, Tucson, 7, 15.

[8] Perim, E., et al. (2016) Spectral Descriptors for Bulk Metallic Glasses Based on the Thermodynamics of Competing Crystalline Phases. Nature Communications, 7, 12315. https://doi.org/10.1038/ncomms12315

[9] Rappaz, M. and Gandin, C.A. (1993) Probabilistic Modelling of Microstructure Formation in Solidification Processes. Acta Metallurgica et Materialia, 41, 345-360. https://doi.org/10.1016/0956-7151(93)90065-Z

[10] Zhang, J., et al. (2013) Probabilistic Simulation of Solidification Microstructure Evolution during Laser-Based Metal Deposition. Proceedings of 2013 Annual International Solid Freeform Fabrication Symposium-An Additive Manufacturing Conference, Austin, August 2013, 739-748. http://scholarsmine.mst.edu/mec aereng facwork/676/

[11] Zhou, X., et al. (2016) Simulation of Microstructure Evolution during Hybrid Deposition and Micro-Rolling Process. Journal of Materials Science, 51, 6735-6749. https://doi.org/10.1007/s10853-016-9961-0

[12] Chen, Y., Zhou, C. and Lao, J. (2011) A Layerless Additive Manufacturing Process Based on CNC Accumulation. Rapid Prototyping Journal, 17, 218-227. https://doi.org/10.1108/13552541111124806

[13] Zinoviev, A., et al. (2016) Evolution of Grain Structure during Laser Additive Manufacturing. Simulation by a Cellular Automata Method. Materials \& Design, 106, 321-329. https://doi.org/10.1016/j.matdes.2016.05.125

[14] Wang, Z.-J., et al. (2014) Simulation of Microstructure during Laser Rapid Forming Solidification Based on Cellular Automaton. Mathematical Problems in Engineering, 2014, 9. https://doi.org/10.1155/2014/627528

[15] Tian, F., Li, Z. and Song, J. (2016) Solidification of Laser Deposition Shaping for TC4 Alloy Based on Cellular Automation. Journal of Alloys and Compounds, 676, 542-550. https://doi.org/10.1016/j.jallcom.2016.03.204

[16] Lindgren, L.-E., et al. (2016) Simulation of Additive Manufacturing Using Coupled Constitutive and Microstructure Models. Additive Manufacturing, 12, 144-158.

[17] Markl, M. and Körner, C. (2016) Multiscale Modeling of Powder Bed-Based Additive Manufacturing. Annual Review of Materials Research, 46, 93-123. https://doi.org/10.1146/annurev-matsci-070115-032158

[18] Fan, Z., et al. (2007) Numerical Simulation of the Evolution of Solidification Microstructure in Laser Deposition. Proceedings of the 18 th Annual Solid Freeform Fabrication Symposium, Austin, August 2007, 256-265.

http://scholarsmine.mst.edu/mec aereng facwork/671/

[19] Zhu, M.F., Lee, S.Y. and Hong, C.P. (2004) Modified Cellular Automaton Model for the Prediction of Dendritic Growth with Melt Convection. Physical Review E, 69, Article ID: 061610. https://doi.org/10.1103/PhysRevE.69.061610

[20] Li, H.Q., Yan, J.H. and Wu, H.J. (2009) Modelling and Simulation of Bulk Metallic Glass Production Process with Suction Casting. Materials Science and Technology, 25, 425-431. https://doi.org/10.1179/174328408X270248 
[21] Browne, D.J., Kovacs, Z. and Mirihanage, W.U. (2009) Comparison of Nucleation and Growth Mechanisms in Alloy Solidification to Those in Metallic Glass Crystallisation-Relevance to Modeling. Transactions of the Indian Institute of Metals, 62, 409-412. https://doi.org/10.1007/s12666-009-0055-4

[22] Wang, C.Y. and Beckermann, C. (1993) A Multiphase Solute Diffusion Model for Dendritic Alloy Solidification. Metallurgical and Materials Transactions A, 24, 2787-2802. https://doi.org/10.1007/BF02659502

[23] Wang, W., Lee, P.D. and McLean, M. (2003) A Model of Solidification Microstructures in Nickel-Based Superalloys: Predicting Primary Dendrite Spacing Selection. Acta Materialia, 51, 2971-2987. https://doi.org/10.1016/S1359-6454(03)00110-1

[24] Wei, Y.H., et al. (2007) Numerical Simulation of Columnar Dendritic Grain Growth during Weld Solidification Process. Science and Technology of Welding and Joining, 12, 138-146. https://doi.org/10.1179/174329307X164427

[25] Nastac, L. (1999) Numerical Modeling of Solidification Morphologies and Segregation Patterns in Cast Dendritic Alloys. Acta Materialia, 47, 4253-4262. https://doi.org/10.1016/S1359-6454(99)00325-0

[26] Gu, C., et al. (2017) A Three-Dimensional Cellular Automaton Model of Dendrite Growth with Stochastic Orientation during the Solidification in the Molten Pool of Binary Alloy. Science and Technology of Welding and Joining, 22, 47-58. https://doi.org/10.1080/13621718.2016.1183962

[27] Dezfoli, A.R.A., et al. (2017) Determination and Controlling of Grain Structure of Metals after Laser Incidence: Theoretical Approach. Scientific Reports, 7, Article ID: 41527. https://doi.org/10.1038/srep41527

[28] Zhu, M.F. and Hong, C.P. (2002) A Three Dimensional Modified Cellular Automaton Model for the Prediction of Solidification Microstructures. ISIJ International, 42, 520-526. 